\title{
Experimental Verification of Semiconductor Diode Aging Based on Thermal Analyzes and Numerical Methods
}

\author{
K. SOKÓє ${ }^{a, *}, \mathrm{P} . \mathrm{PTAK}^{a}$ AND J. PODEŠVA ${ }^{b}$ \\ ${ }^{a}$ Częstochowa University of Technology, 42-201 Częstochowa, Poland \\ ${ }^{b}$ VSB - Technical University of Ostrava, 70800 Ostrava-Poruba, Czech Republic \\ Doi: 10.12693/APhysPolA.139.517 \\ *e-mail: sokol@imipkm.pcz.pl
}

\begin{abstract}
Many electronic systems, such as computers and telephones, have a short service life, typically between two and five years. However, there are applications such as cars or airplanes that can have service life of 15 years or more. While product maintenance is usually practiced for mechanical components, it is difficult to find information in the technical literature as to whether the maintenance of electronic circuits can be justified in the case of very long-term use and whether it is possible at all. The approach to the behavior of components during failure is the closest to describing the "life time of electronic products." The described product life models are useful in cases where this life is limited by predictable physical mechanisms. These models perform well, for example, for solder joint fatigue caused by thermal cycling. This article describes an experimental verification of the aging of semiconductor diodes in order to determine their technical condition and current characteristics after 15 years of use. The obtained results of experimental research were compared with those from numerical simulations. On the basis of the obtained data, it was determined whether the aging process of the analyzed components takes place and how it affects their operation. Numerical models were created on the basis of the Coffin-Manson and Arrhenius equations.
\end{abstract}

topics: thermal analysis, experimental test, diode, WCA test

\section{Introduction}

Electronics is the field of science that deals with the use of the phenomenon of electron motion in vacuum, gases and solids. These phenomena are the basis for the functioning of electronic systems, especially in semiconductor systems. Over the years, discovering the applications of this phenomenon has led to the fact that today we are surrounded by the enormity of electronic devices. A generally understood electronic system is a set of cooperating passive and active components that meet strictly defined requirements. The most common and effective method of electronic circuit verification is the worst-case analysis (WCA). During the early stage of the project, it is crucial for the engineers to define margins resulting from customer requirements and technical documentation of the components. More information on the WCA analysis can be found in [1-5].

\section{Analyzed circuit}

The analyzed semiconductor diode ultimately works in the temperature sensor module, transmitting information to the microcontroller about the operating temperature of the motor at which this

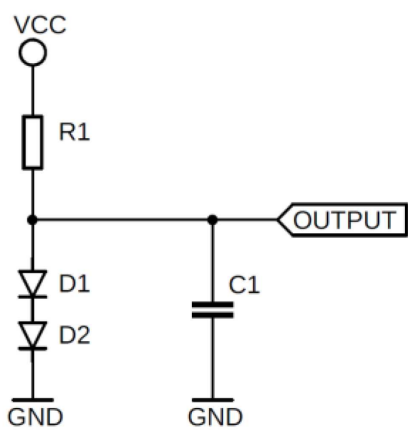

Fig. 1. Circuit diagram.

module is installed. The diagram of the sensor is shown in Fig. 1. The system consists of an actuator - two semiconductor diodes connected in series (in one housing, working in the system as a temperature sensor), a resistor limiting the current consumption and a capacitor that filters the output voltage. Due to linear temperature, coefficient diodes are commonly used as a thermometer. To conduct laboratory tests allowing for experimental verification of the aging process of semiconductor diodes, PCBs specially designed for this purpose were prepared. 


\section{Test setup}

This allows one to measure the voltages of large series of diodes simultaneously. Moreover, the copper traces were designed so that their length was approximately identical for each of the measured diodes. The analyzed diode models are presented in Table I.

The measurement during the test consists in determining the voltage on the diode pairs, ignoring differences resulting from voltage drops on the tracks and wires connecting the diode pad with the measuring device.

Voltage readings were made for nine different temperatures ranging from $-40{ }^{\circ} \mathrm{C}$ to $+150^{\circ} \mathrm{C}$ and for fifteen current levels ranging from $100 \mu \mathrm{A}$ to $1500 \mu \mathrm{A}$. The exactly defined levels of currents were obtained in the measuring system through current excitation, set by means of the DC Load programmed for automatic operation, allowing for an effective measurement of large series of components. During the tests, the diode voltage was measured with a scopecorder. It was decided to use this measuring device due to its modularity, multi-channels and the ability to record waveforms over time.

\section{Development test's results and conclusions}

The measurements were conducted before and after the diodes were subjected to an accelerated high temperature aging test. It should be noted that the tests were carried out in a repeatable manner, so that the behavior of each of the measured diodes is known exactly.

An example of the obtained results for standard deviations is presented in Table II. On its basis, it can be noticed that the standard deviation as a whole increases with the increase of the flowing current and decreases with the increase of the ambient temperature. The obtained values of standard
Analyzed types of diodes.

TABLE I

\begin{tabular}{l|l|l}
\hline \hline Supplier & \multicolumn{1}{|c|}{ Series } & \multicolumn{1}{c}{ Package } \\
\hline Vishay & BAV99 & SOT23 \\
NXP & BAV99W & SOT323 \\
Onsemi & BAV99W & SOT323
\end{tabular}

deviations for the temperature of $25^{\circ} \mathrm{C}$ (observed with LEDs of all manufacturers) — different from the rest of the results - are for sure the result of a measurement error. It had been the result of the human factor which, due to the effects imperceptible during the test, was detected only during a further data analysis. Due to the unnoticeable standard deviations during the test and the fact that the calculations were carried out after the entire test, it is impossible to repeat the test on the same series of diodes. Comparing the obtained data with the manufacturer's data and the overall view of the test performed, it is recognized that the test is as important and reliable as possible, but the results obtained at this temperature of $25^{\circ} \mathrm{C}$ should be ignored in creating final conclusions.

In the next stage, analogous diode measurements were carried out after the accelerated aging simulation was performed, in accordance with the obtained simulation duration of $357.4 \mathrm{~h}$. Table III shows the mean standard deviation obtained for the measurements after the final test. It can be immediately noticed that after the test, the standard deviations for each analyzed condition changed and that the tendency of the diode forward voltage change did not take place in one direction. The standard deviations obtained in the final test are lower than the original ones. This may be due to the fact that the components, as a function of time, tend to a certain limit, a fixed value, at which they function for the rest of their life cycle. This can be compared to some extent to a bathtub curve. Figure 2 shows an example of the results obtained for one of the analyzed producers at a temperature of $0^{\circ} \mathrm{C}$ and in the range of $100 \mu \mathrm{A}-1500 \mu \mathrm{A}$.

Pre-test, the obtained standard deviation results $[\mathrm{mV}]-$ NXP.

TABLE II

\begin{tabular}{c|c|c|c|c|c|c|c|c|c|c|c|c|c|c|c}
\hline \hline \multirow{2}{*}{$T\left[{ }^{\circ} \mathrm{C}\right]$} & \multicolumn{10}{c}{$I[\mu \mathrm{A}]$} \\
\cline { 2 - 12 } & 100 & 200 & 300 & 400 & 500 & 600 & 700 & 800 & 900 & 1000 & 1100 & 1200 & 1300 & 1400 & 1500 \\
\hline-40 & 3.7 & 4.3 & 4.5 & 4.7 & 4.8 & 4.9 & 4.9 & 5.0 & 5.0 & 5.1 & 5.1 & 5.2 & 5.2 & 5.2 & 5.2 \\
-20 & 3.0 & 3.9 & 4.4 & 4.7 & 5.0 & 5.2 & 5.3 & 5.4 & 5.5 & 5.6 & 5.7 & 5.7 & 5.8 & 5.9 & 6.5 \\
0 & 4.0 & 3.7 & 4.1 & 4.4 & 4.7 & 5.0 & 5.2 & 5.3 & 5.5 & 5.6 & 5.8 & 5.9 & 6.0 & 6.1 & 6.6 \\
25 & 34.9 & 13.3 & 8.7 & 6.9 & 6.2 & 5.7 & 5.5 & 5.4 & 5.3 & 5.4 & 5.4 & 5.4 & 5.4 & 5.5 & 6.8 \\
50 & 2.2 & 2.0 & 2.1 & 2.2 & 2.3 & 2.5 & 2.6 & 2.8 & 2.9 & 3.0 & 3.1 & 3.2 & 3.4 & 3.5 & 4.6 \\
75 & 2.2 & 2.1 & 1.9 & 1.9 & 1.8 & 1.8 & 1.9 & 1.9 & 2.0 & 2.0 & 2.1 & 2.2 & 2.2 & 2.3 & 2.4 \\
100 & 2.5 & 2.4 & 2.3 & 2.1 & 2.1 & 2.1 & 2.0 & 2.0 & 2.0 & 2.0 & 2.0 & 2.1 & 2.1 & 2.1 & 2.1 \\
125 & 3.0 & 3.1 & 2.8 & 2.7 & 2.7 & 2.6 & 2.6 & 2.5 & 2.5 & 2.5 & 2.5 & 2.5 & 2.5 & 2.5 & 2.5 \\
150 & 3.0 & 3.0 & 2.9 & 2.8 & 2.7 & 2.7 & 2.6 & 2.6 & 2.6 & 2.6 & 2.6 & 2.6 & 2.5 & 2.5 & 2.5
\end{tabular}


Final-test, standard deviation - NXP.

TABLE III

\begin{tabular}{c|c|c|c|c|c|c|c|c|c|c|c|c|c|c|c}
\hline \hline \multirow{2}{*}{$T\left[{ }^{\circ} \mathrm{C}\right]$} & \multicolumn{10}{c|}{$I[\mu \mathrm{A}]$} \\
\cline { 2 - 13 } & 100 & 200 & 300 & 400 & 500 & 600 & 700 & 800 & 900 & 1000 & 1100 & 1200 & 1300 & 1400 & 1500 \\
\hline-40 & 0.97 & 0.97 & 0.69 & 0.55 & 0.47 & 0.42 & 0.39 & 0.36 & 0.34 & 0.32 & 0.30 & 0.30 & 0.29 & 0.28 & 7.80 \\
-20 & 0.87 & 0.89 & 0.64 & 0.53 & 0.45 & 0.41 & 0.37 & 0.35 & 0.33 & 0.32 & 0.31 & 0.31 & 0.30 & 0.29 & 4.98 \\
0 & 3.35 & 2.61 & 2.63 & 2.76 & 2.88 & 2.99 & 3.09 & 3.17 & 3.25 & 3.31 & 3.38 & 3.44 & 3.51 & 3.56 & 4.69 \\
25 & 35.64 & 13.79 & 8.85 & 6.84 & 5.80 & 5.14 & 4.73 & 4.42 & 4.10 & 3.95 & 3.92 & 3.84 & 3.74 & 3.71 & 7.79 \\
50 & 1.26 & 1.15 & 1.09 & 1.07 & 1.07 & 1.06 & 1.06 & 1.06 & 1.04 & 1.04 & 1.03 & 1.04 & 1.02 & 1.01 & 3.63 \\
75 & 0.93 & 0.96 & 0.87 & 0.85 & 0.84 & 0.85 & 0.87 & 0.89 & 0.90 & 0.92 & 0.94 & 0.96 & 0.98 & 1.00 & 2.46 \\
100 & 1.74 & 1.74 & 1.48 & 1.37 & 1.32 & 1.32 & 1.32 & 1.33 & 1.35 & 1.38 & 1.39 & 1.42 & 1.44 & 1.46 & 8.57 \\
125 & 2.50 & 2.15 & 1.88 & 1.77 & 1.74 & 1.72 & 1.71 & 1.70 & 1.68 & 1.66 & 1.66 & 1.62 & 1.60 & 1.59 & 6.83 \\
150 & 4.24 & 4.24 & 3.97 & 3.82 & 3.73 & 3.71 & 3.64 & 3.60 & 3.57 & 3.55 & 3.53 & 3.55 & 3.52 & 3.51 & 13.60
\end{tabular}

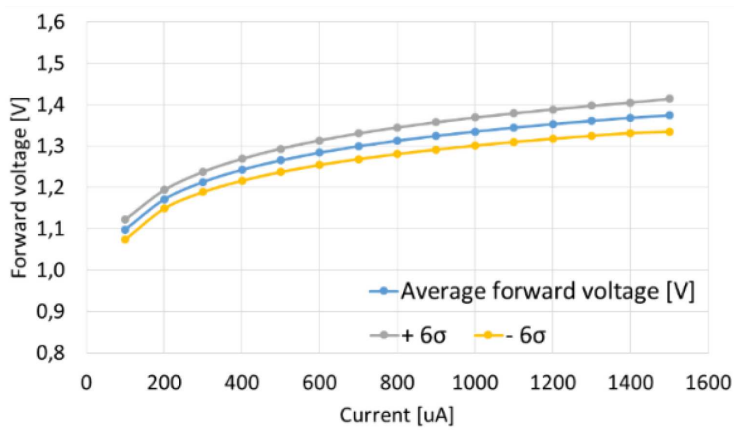

Fig. 2. Example of results of the test $-\operatorname{NXP} 0{ }^{\circ} \mathrm{C}$.

The data in Fig. 3 perform the distribution of the number of diodes as a function of the forward voltage change after the test for each diode model. As can be seen from Fig. 3, most of the diodes measured show no response to the forward voltage change after the test.

It can also be noted that although most of the measurements oscillate around $\Delta=0 \mathrm{mV}$, the trend of changes is rather towards the lower values obtained after the test. Table IV presents a summary of all three manufacturers and the results obtained, respectively, before and after the test, average measurements and standard deviations for the selected case (ambient temperature $-40^{\circ} \mathrm{C}$ and current flowing $1500 \mu \mathrm{A})$. It was decided to choose this selected temperature and current level because of the largest absolute values between the values of the samples.

It can be seen that the values after the test are on average about $3 \mathrm{mV}$ lower for each manufacturer. This may indicate a tendency of the diodes to decrease their values over time, which is also confirmed by the observation of the data in Fig. 3. The standard deviation contained in Table IV indicates a greater dispersion of the obtained results, that is, a wider range of tolerance in which the analyzed series are located. The aging simulation resulted in greater deviations from the mean value.

Further calculations were made to better assess the aging of the semiconductors. The significance of the linear one-dimensional regression function was

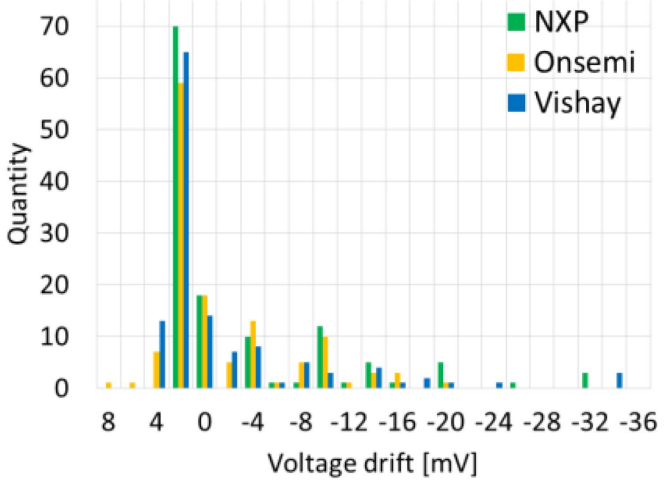

Fig. 3. Distribution of a number of diodes as a function of voltage drift.

investigated. For this purpose, the previously calculated mean value and standard deviations were used. The moment of correlation of the variables from the sample was successively determined with the use of the moment of correlation

$$
m_{x y}=\frac{1}{n} \sum_{i=1}^{n}\left(x_{i}-m_{x}\right)\left(y_{i}-m_{y}\right)
$$

and the linear correlation coefficient

$$
R_{x y}=\frac{\frac{1}{n} \sum_{i=1}^{n}\left(x_{i}-m_{x}\right)\left(y_{i}-m_{y}\right)}{\sqrt{\sum_{i=1}^{n}\left(x_{i}-m_{x}\right)^{2}} \sqrt{\sum_{i=1}^{n}\left(y_{i}-m_{y}\right)^{2}}},
$$

where the number of test samples is $n, x_{i}$ - the value of the next tested sample, $m_{x}$ - the average value of the next tested sample, $y_{i}$ - the value of the next sample finally tested, and $m_{y}$ - the average value of the next samples finally tested.

The linear correlation coefficient determines the degree of linear dependence between the variables. The value of the factor -1 or 1 proves that there is a strict linear correlation between the variables. However, when the coefficient is equal to or its value is close to zero, the analyzed variables are uncorrelated. The closer the absolute value of the coefficient is to 1 , the stronger the correlation between the variables [6]. 
TABLE IV

Forward voltage before and after the test at $-40{ }^{\circ} \mathrm{C}$ and current flowing $1500 \mu \mathrm{A}$.

\begin{tabular}{l|l|c|c|c}
\hline \hline \multirow{2}{*}{} & \multicolumn{2}{|c|}{ Avg. voltage [V] } & \multicolumn{2}{c}{ Std. dev. [mV] } \\
\cline { 2 - 5 } & Before & After & Before & After \\
\hline NXP & 1.5276 & 1.5233 & 5.239 & 7.8031 \\
Onsemi & 1.4841 & 1.4816 & 1.702 & 5.2859 \\
Vishay & 1.5130 & 1.5103 & 1.207 & 7.5302
\end{tabular}

Summary of the analysis of correlation. TABLE V

\begin{tabular}{l|c|c|c}
\hline \hline & $\begin{array}{c}\text { Correlation } \\
\text { coefficient }\end{array}$ & $t_{-}{ }^{\alpha}$ & $t$ coefficient \\
\hline NXP & 0.66 & 2.576 & 9.8 \\
Onsemi & 0.29 & 2.576 & 3.4 \\
Vishay & 0.04 & 2.576 & 0.5 \\
Total avg. & 0.33 & 2.576 & 4.6
\end{tabular}

As shown in Table $\mathrm{V}$, the total averaged correlation coefficient for all tested diodes is 0.33 , which means, from an analytical point of view, that the correlation of diode conduction voltage changes as a function of time is very weak. The NXP diodes reached the highest value here $(0.66)$, but it is not close enough to 1 to speak of a noticeable aging of these diodes. To confirm the conclusion about the weak correlation, we have calculated

$$
t=\frac{R_{x y}}{\sqrt{1-R_{x y}^{2}}} \sqrt{n-2} .
$$

Using Student's $t$-distribution for $\infty$ degrees of freedom and $\alpha=0.01$ ( $\infty$ degrees of freedom because one uses more than 120 elements for the test and $\alpha=0.01$ because the diode aging is assumed to be negligible), one can read from Student's $t$-tables $t_{\_} \alpha=2.576$. The calculated $t$ factor for the analyzed series of diodes is 4.6. If $|t| \geq t \_\alpha$, the hypothesis about the lack of correlation between the analyzed variables (time and forward voltage) should be rejected.
However, when $|t| \leq t \_\alpha$, there is no reason to reject the assumed hypothesis that the variables are related. On the basis of the results of the $t$ coefficient, it can be concluded that the hypothesis about the influence of time on changes in diode voltage conduction is justified in looking at the results in general, which stems from $|t| \geq t \_\alpha$. It should be noted, however, that Vishay diodes qualify for rejecting the hypothesis about the correlation of diode forward voltage versus time as we see in Table V.

\section{Conclusions}

Summarizing the conducted test simulating the aging of semiconductor diodes, it can be concluded that there is a correlation between the lifetime of the diode and the forward voltage. Based on a series of calculations, it can be stated that the observed correlation is weak, which in less critical applications may be the basis for omitting this parameter as a component of the WCA analysis. Each of the tested manufacturers finally fared similarly to the rest, while the Vishay LEDs had slightly the best results.

\section{References}

[1] R. Chapman, A. Burns, A.J. Wellings, Real-Time Syst. 11, 145 (1996).

[2] A. Ermedahl, J. Gustafsson Proc. of Europar'97.

[3] Y. Li, S. Malik, A. Wolf, in: Proc. of the 17th IEEE Real-Time Systems Symposium, Washington, IEEE, 1996, p. 254.

[4] R. White, F. Mueller, C. Healy, D. Whalley, M. Harmon, IEEE Real-Time Technology and Applications Symposium, Montreal, IEEE, 1997, p. 192.

[5] K. Sokół, P. Ptak, Acta Phys. Pol. A 138, 291 (2020).

[6] E. Majchrzak, B. Mochnacki, Metody numeryczne, Wydawnictwo Politechniki Śląskiej, Gliwice 2004 (in Polish). 